\title{
Evaluating the appropriateness of antibiotic treatment of tonsillitis during COVID-19 in the north Wales primary healthcare setting: a preliminary report
}

\author{
Authors: Chunhei Li, ${ }^{\mathrm{A}}$ Anna Morris, ${ }^{\mathrm{A}}$ Caitlin Ong, ${ }^{\mathrm{A}}$ Ahsan Ashfaq, ${ }^{\mathrm{A}}$ Rikesh Jagatia ${ }^{\mathrm{A}}$ and Isobel Woollons ${ }^{\mathrm{A}}$
}

\begin{tabular}{|c|c|c|c|c|}
\hline Baseline & Total & Face-to-face group & Remote group & $p$ value \\
\hline Age, years, median (range) & $21(1-85)$ & $18(1-51)$ & $23(5-85)$ & 0.145 \\
\hline Male:female ratio & $0.85: 1$ & $0.43: 1$ & $1.67: 1$ & 0.0973 \\
\hline Antibiotic given & & & & 0.88 \\
\hline Yes & $83.1 \%$ & $87.5 \%$ & $83.33 \%$ & \\
\hline No & $16.9 \%$ & $12.5 \%$ & $16.7 \%$ & \\
\hline FeverPain or CENTOR scoring system used & & & & 0.2208 \\
\hline Yes & $13.8 \%$ & $0 \%$ & $17.39 \%$ & \\
\hline No & $86.2 \%$ & $100 \%$ & $82.61 \%$ & \\
\hline Parameters recorded in the notes $(\mathrm{Y} / \mathrm{N})$ & & & & $0.008115^{*}$ \\
\hline Yes & $66.2 \%$ & $93.75 \%$ & $48.82 \%$ & \\
\hline No & $33.8 \%$ & $6.25 \%$ & $52.17 \%$ & \\
\hline $\begin{array}{l}\text { Does the FeverPAIN score recommend antibiotic } \\
\text { prescription? }\end{array}$ & & & & 0.99 \\
\hline Yes & $44.6 \%$ & $73.33 \%$ & $39.13 \%$ & \\
\hline No & $23.07 \%$ & $26.67 \%$ & $43.47 \%$ & \\
\hline Cannot work out & $32.3 \%$ & $0 \%$ & $39.1 \%$ & \\
\hline
\end{tabular}

*Significant difference between the groups.

\section{Background}

Acute tonsillitis is a common cause of sore throat that is frequently seen in primary care. Currently, NICE guidance recommends either using the CENTOR or FeverPAIN scoring criteria to risk-stratify patients with tonsillitis and assess the need for an antibiotic prescription. ${ }^{1}$ During the COVID-19 pandemic, GP consultations largely moved from face-toface to remote consultations. This potentially makes it more challenging for clinicians to use scoring systems.

This study aims to evaluate the appropriateness of antibiotic treatment of tonsillitis during the COVID-19 pandemic in the primary care setting. ${ }^{2-5}$ The three main objectives of this study

Author: ${ }^{A}$ Cardiff University, Cardiff, UK; all authors contributed equally were to determine the number of cases of acute tonsillitis that were treated with antibiotics, to assess whether any scoring criteria were used to guide the antibiotic prescription or whether any parameters were documented in the notes, and to assess any differences between remote consultations and face-to-face consultations.

\section{Method}

This is a multicentre observational study performed in five general practice surgeries in north-west Wales. Computer records of patients with a confirmed diagnosis of acute tonsillitis were retrospectively reviewed from March 2020 until the present. A six-item questionnaire was produced and used to standardise the data collection across sites. For any encounters that were not awarded a score at the time of consultation, but where all parameters were recorded, a score was awarded to allow for analysis of antibiotic prescription. Chi-squared and Mann-Whitney 
tests were used to compare differences between groups. A p value of 0.05 was set to be statistically significant in this study.

\section{Results}

A total of 155 patients were identified and enrolled in this study across five different GP practices. This preliminary report contained 65 patients that had completed data collection and input for analysis. During this period, only $13.8 \%$ used either FeverPAIN or CENTOR scoring system, while $86.2 \%$ did not use an objective scoring system. $48.8 \%$ of remote consultations and $93.8 \%$ of face-to-face consultations recorded all the parameters required to calculate a FeverPAIN score. This difference was shown to be statistically significant $(p=0.0081)$. There was no statistically significant difference in the prescription of antibiotics between the remote and face-to-face consultations $(\mathrm{p}=0.88)$.

$83.1 \%$ of patients were prescribed antibiotics despite only $44.6 \%$ of scores calculated recommending the use of antibiotics. However, nearly a third of consultations $(32.3 \%)$ did not have the parameters recorded to allow a retrospective score to be calculated. There was no statistically significant difference between remote and face-to-face consultations as to whether the scores recommended the use of antibiotics or not $(\mathrm{p}=0.99)$. The results are summarised in Table 1.

\section{Discussion}

Our preliminary report has demonstrated that general practitioners using remote consultation did not differ significantly in an antibiotic prescription, compared to face-to-face consultation. This finding encouragingly suggests that remote consultation is as effective as face-to-face consultation concerning antibiotic prescribing in tonsillitis. Interestingly, we noticed more missing data in the notes associated with remote consultation. This could be attributed to the difficulty of performing objective assessments via remote consultation. We await a further 85 patients' results to arrive for further analysis.

\section{Conflicts of interest}

None declared.

\section{References}

1 National institute for Health and Care Excellence. Sore throat (acute): antimicrobial prescribing: NICE Guidance [NG84]. NICE, 2018. www.nice.org.uk/guidance/ng84 [Accessed 14 October 2020].

2 Spinks A, Glasziou PP, Del Mar CB et al. Antibiotics for sore throat. Cochrane Database Syst Rev 2013;11:CD000023.

3 Windfuhr JP, Toepfner N, Steffen G et al. Clinical practice guideline: tonsillitis I. Diagnostics and nonsurgical management. Eur Arch Otorhinolaryngol 2016;273:973-87.

4 Dolk F, Pouwels K, Smith D et al. Antibiotics in primary care in England: which antibiotics are prescribed and for which conditions? J Antimicrob Chemother 2018;73(suppl_2):ii2-10.

5 Fine A, Nizet V, Mandl K. 2012. Large-scale validation of the Centor and McIsaac scores to predict Group A streptococcal pharyngitis. Arch Intern Med 2012;172:847-52. 